Pacific

Journal of

Mathematics

UNIQUENESS AND A PRIORI ESTIMATES FOR SOME NONLINEAR ELLIPTIC NEUMANN EQUATIONS IN $\mathbb{R}^{3}$

Juncheng Wei And Xingwang Xu 


\title{
UNIQUENESS AND A PRIORI ESTIMATES FOR SOME NONLINEAR ELLIPTIC NEUMANN EQUATIONS IN $\mathbb{R}^{3}$
}

\author{
JUNCHENG WEI AND XINGWANG XU
}

\begin{abstract}
Under some conditions on $f(u)$, we show that for $\lambda$ small and $\Omega \subset \mathbb{R}^{3}$ convex, the only solution to the elliptic equation $\Delta u-\lambda u+f(u)=0$ in $\Omega$, with $u>0$ in $\Omega$ and $\partial u / \partial v=0$ on $\partial \Omega$, is constant.
\end{abstract}

\section{Introduction}

We consider the nonlinear elliptic equation

$$
\left\{\begin{array}{l}
\Delta u-\lambda u+f(u)=0 \text { in } \Omega, \\
u>0 \text { in } \Omega, \frac{\partial u}{\partial v}=0 \text { on } \partial \Omega,
\end{array}\right.
$$

where $\Omega$ is a smooth and bounded domain in $\mathbb{R}^{3}$, the function $f$ lies in $C^{1+\theta}$ for some $0<\theta<1$, and $v$ is the unit outer normal vector field at $\partial \Omega$. We suppose that $f(u)$ can be written as $f(u)=u^{5}(1+\rho(u))$, with $\rho^{\prime}(u) \leq 0,0 \leq u^{5} \rho(u) \leq$ $C \sum_{i=1}^{K} u^{p_{i}}$ for some $1<p_{i} \leq \frac{13}{3}$ and some constant $C>0$. A typical example for $f(u)$ is the function

$$
f(u)=u^{5}+\sum_{i=1}^{K} a_{i} u^{p_{i}}, \quad \text { with } 1<p_{i} \leq \frac{13}{3} \text { and } a_{i} \geq 0 \text { for } i=1, \ldots, K .
$$

Our main result is the following.

Theorem 1. Suppose that $\Omega \subset \mathbb{R}^{3}$ is convex. Then there exist $\lambda_{0}>0$ and $C>0$ such that for $\lambda<\lambda_{0}$ we have

$$
u \leq C \lambda^{1 / 4}
$$

for all solutions $u$ of (1.1), where $C$ is independent of $\lambda$.

As a consequence, $u \equiv C_{\lambda}$ for some constant $C_{\lambda}$.

MSC2003: primary 35J60, 35B20; secondary 53C21.

Keywords: critical Sobolev exponent, Neumann equation, nonlinear elliptic PDEs.

Wei's research is supported by an Earmarked Grant from RGC of Hong Kong. Xu's research is supported by research grant No. R-146-000-008-112. This project was initiated when Xu was visiting the Department of Mathematics of the Chinese University of Hong Kong. He thanks the department for its hospitality and support. 
Remark. The restriction that $u^{5} \rho(u) \leq C \sum_{i=1}^{K} u^{p_{i}}$ for $p_{i} \leq \frac{13}{3}$ just reflects a technical difficulty. We believe that Theorem 1 also holds under the weaker assumption $0 \leq u^{5} \rho(u) \leq C \sum_{i=1}^{K} u^{p_{i}}$ for $1<p_{i}<5$.

The proof of Theorem 1 is a direct consequence of integration by parts. It also gives a new and rather simple derivation of the following theorem.

Theorem 2. Suppose that $\Omega \subset \mathbb{R}^{3}$ is convex and $f(u)=u^{5}$. Then for $\lambda$ small, $u=\lambda^{1 / 4}$ is the unique solution of (1.1).

Theorem 2 is a special case of Lin and Ni's conjecture [Lin and Ni 1988]. The conjecture says that for $\lambda$ small and $f(u)=u^{p}, p>1$, the problem (1.1) admits only constant solutions. (Problem (1.1) with $f(u)=u^{p}$ arises in the study of steady-state solutions of the model of Keller and Segel [1970] in chemotaxis and the Gierer-Meinhardt system [1972] in pattern formation. For more background on (1.1), see [Ni 1998].)

Lin and Ni proved their conjecture in the case when $p$ is subcritical. Zhu [1999] proved the conjecture for three-dimensional convex domains (the same class of domains as Theorem 2) by using a very complicated blow-up analysis. Zhu's proof is by contradiction and thus indirect. It is unclear if Zhu's proof can be generalized to other functions $f(u)$. Our proof is much simpler and elementary. In fact since our proof is direct, it can yield an explicit value for the number $\lambda_{0}$ in Theorem 1 . We remark that when $\Omega=B_{R}(0)$ and $u$ is radial, similar results were proved by Adimurthi and Yadava [1993].

In higher dimensions, the Lin-Ni conjecture may be wrong. Counterexamples in the radial case are given in [Adimurthi and Yadava 1991; 1997]. For the nonradial case, see [Gui and Wei 2005].

We use an idea developed by Chang, Gursky and Yang in the study of threedimensional prescribed scalar curvature problem [Chang et al. 1993]. Our starting point is to write equation (1.1) as

$$
\Delta u-\lambda u+f(u)=\Delta u+R(u) u^{5},
$$

where $R(u)=1+\rho(u)-\lambda u^{-4}$. Thus if we introduce the conformal transformation $g=u^{4} g_{0}$, where $g_{0}$ is the usual Euclid metric, $R$ becomes the scalar curvature in the new metric.

We first make some preliminary notes. (Throughout this paper, $C$ will denote different constants independent of $\lambda$.)

From equation (1.1), we see that

$$
\int_{\Omega} u^{5} \leq \int_{\Omega} f(u)=\lambda \int_{\Omega} u \leq C \lambda\left(\int_{\Omega} u^{5}\right)^{1 / 5},
$$


which implies that

$$
\int_{\Omega} u^{5} \leq C \lambda^{5 / 4}, \quad \int_{\Omega} u \leq \lambda^{1 / 4}
$$

and thus

$$
u_{\min }:=\min _{x \in \bar{\Omega}} u(x) \leq C \lambda^{1 / 4} .
$$

From (1.2), we also obtain that

$$
\int_{\Omega} u^{q} \leq C \lambda^{q / 4} \text { for all } 0<q \leq 5 .
$$

Next we need a well-known fact found in [Lions 1982, Appendix 1, Lemma 5], for example: if $\Omega$ is convex and $\partial u / \partial v=0$ on $\partial \Omega$, then

$$
\frac{\partial}{\partial v}|\nabla u|^{2} \leq 0 \quad \text { on } \partial \Omega
$$

\section{Proof of Theorem 1}

Let $g_{0}$ be the usual Euclidean metric and $g=u^{4} g_{0}$. In the new metric, we consider the trace-free Ricci tensor $B$. In a local coordinate system, we may write

$$
B_{i j}=-u^{-2}\left(\left(u^{2}\right)_{g, i j}-\frac{1}{3}\left(\Delta_{g} u^{2}\right) g_{i j}\right) .
$$

(Here the covariant derivatives are taken with respect to $g$, not $g_{0}$.)

We first obtain an integral estimate for $\int_{\Omega}|B|^{3 / 2} d V_{g}$. (Here $d V_{g}$ denotes integration with respect to the new metric $g$. It is easy to see that $d V_{g}=u^{6} d x$.) To this end, we consider

$$
\begin{aligned}
\int_{\Omega}|B|^{2} u^{2} d V_{g} & =\int_{\Omega} g^{i k} g^{j l} B_{i j} B_{k l} u^{2} d V_{g} \\
& =-\int_{\Omega}\left(u^{2}\right)_{g, i j} B_{k l} g^{i k} g^{j l} d V_{g}+\frac{1}{3} \int_{\Omega}\left(\Delta_{g} u^{2}\right) g_{i j} g^{i k} g^{j l} B_{k l} d V_{g},
\end{aligned}
$$

where the second term vanishes because $B$ is trace-free.

Now using integration by parts, together with the Neumann boundary condition, we have

$$
\int_{\Omega}|B|^{2} u^{2} d V_{g}=\int_{\Omega}\left(u^{2}\right)_{g, i} B_{k l ; j} g^{i k} g^{j l} d V_{g}+\int_{\partial \Omega} \frac{\partial}{\partial v}|\nabla u|^{2} d \sigma_{g},
$$

where $d \sigma_{g}$ is the surface element in the new metric $g$.

Using the contracted second Bianchi identity and (1.5) we obtain 


$$
\begin{aligned}
\int_{\Omega}|B|^{2} u^{2} d V_{g} & \leq \frac{1}{6} \int_{\Omega}\left(u^{2}\right)_{g, i} R_{, k} g^{i j k} d V_{g} \\
& =\frac{1}{6} \int_{\Omega}\left\langle\nabla_{g}\left(u^{2}\right), \nabla_{g} R\right\rangle d V_{g}=\frac{1}{12} \int_{\Omega}\left\langle\nabla\left(u^{4}\right), \nabla R\right\rangle d x \\
& =\frac{1}{12} \int_{\Omega} \nabla u^{4} \nabla\left(1+\rho(u)-\lambda u^{-4}\right) \\
& \leq \frac{4}{3} \lambda \int_{\Omega} u^{-2}|\nabla u|^{2} d x \quad\left(\text { since } \rho^{\prime}(u) \leq 0\right) \\
& =\frac{4}{3} \lambda \int_{\Omega} u^{-1} \Delta u d x=\frac{4}{3} \lambda \int_{\Omega} u^{-1}(\lambda u-f(u)) \leq C \lambda^{2} .
\end{aligned}
$$

Thus we obtain the following key estimate (here we need $\Omega \subset \mathbb{R}^{3}$ )

$$
\int_{\Omega}|B|^{3 / 2} d V_{g} \leq\left(\int_{\Omega}|B|^{2} u^{2} d V_{g}\right)^{3 / 4}\left(\int_{\Omega} u^{-6} d V_{g}\right)^{1 / 4} \leq C \lambda^{3 / 2} .
$$

Next we estimate $\int_{\Omega}\left|\nabla u^{-2}\right|^{6} d x$. Let $v=1 / u$. Recall the Bochner identity:

$$
\Delta_{g}\left|\nabla_{g} v\right|^{2}=2\left|\nabla_{g}^{2} v\right|^{2}+2\left\langle\nabla_{g} v, \nabla_{g}\left(\Delta_{g} v\right)\right\rangle+2 \operatorname{Ric}_{g}\left(\nabla_{g} v, \nabla_{g} v\right) .
$$

If $\Omega$ is convex, $\int_{\Omega} \Delta_{g}\left|\nabla_{g} v\right|^{2} d V_{g} \leq 0$ by (1.5). Integrating both sides of (2.7) and using the divergence theorem, we get

$$
\int_{\Omega}\left|\nabla_{g}^{2} v\right|^{2} d V_{g} \leq \int_{\Omega}\left(\Delta_{g} v\right)^{2} d V_{g}+\int_{\Omega}|\operatorname{Ric}|\left|\nabla_{g} v\right|^{2} d V_{g}
$$

Recall the Sobolev inequality in $\mathbb{R}^{3}$

$$
\left(\int_{\Omega} \psi^{6} d V_{g}\right)^{1 / 3} \leq C \int_{\Omega}\left|\nabla_{g} \psi\right|^{2} d V_{g}+C \int_{\Omega} \psi^{2} d V_{g}
$$

Take $\psi=\left|\nabla_{g} v\right|$. Then we obtain

$$
\begin{aligned}
& \left(\int_{\Omega}\left|\nabla_{g} v\right|^{6} d V_{g}\right)^{1 / 3} \\
& \quad \leq\left.\left. C \int_{\Omega}\left|\nabla_{g}\right| \nabla_{g} v\right|^{2}\left|+C \int_{\Omega}\right| \nabla_{g} v\right|^{2} \\
& \quad \leq C \int_{\Omega}\left|\nabla_{g}^{2} v\right|^{2} d V_{g}+C \int_{\Omega}\left|\nabla_{g} v\right|^{2} d V_{g} \quad \text { (by Kato's inequality) } \\
& \quad \leq C \int_{\Omega}\left|\Delta_{g} v\right|^{2} d V_{g}+C \int_{\Omega}|\operatorname{Ric}|\left|\nabla_{g} v\right|^{2} d V_{g}+C \int_{\Omega}\left|\nabla_{g} v\right|^{2} d V_{g} \\
& \quad \leq C \int_{\Omega}\left|\Delta_{g} v\right|^{2} d V_{g}+C \int_{\Omega}|B|\left|\nabla_{g} v\right|^{2} d V_{g}+C \int_{\Omega}|R|\left|\nabla_{g} v\right|^{2} d V_{g}+C \int_{\Omega}\left|\nabla_{g} v\right|^{2} d V_{g},
\end{aligned}
$$


where we have used the fact that $|\operatorname{Ric}| \leq|B|+\frac{1}{3}|R|$.

We estimate each term on the last two lines of (2.8).

Recall that $g_{0}=u^{-4} g=v^{4} g$. The scalar curvature $R_{0}$ with respect to $g_{0}$ is 0 . So

$$
\begin{aligned}
\Delta_{g} v & =\left(\Delta_{g}+\frac{1}{8} R\right) v-\frac{1}{8} R v=-\frac{1}{8} R_{0} v^{5}-\frac{1}{8} R v \\
& =-\frac{1}{8} R v=-\frac{1}{8}\left(1+\rho(u)-\lambda u^{-4}\right) u^{-1} .
\end{aligned}
$$

Therefore

$$
\left(\Delta_{g} v\right)^{2} \leq C\left(u^{-2}+u^{-2} \rho^{2}(u)+\lambda^{2} u^{-10}\right) \leq C\left(u^{-2}+\sum_{i=1}^{K} u^{2 p_{i}-12}+\lambda^{2} u^{-10}\right)
$$

and

$\int_{\Omega}\left(\Delta_{g} v\right)^{2} d V_{g} \leq C+C \sum_{i=1}^{K} \int_{\Omega} u^{2 p_{i}-6} d x+C \lambda^{2} \int_{\Omega} u^{-4} d x \leq C\left(1+u_{\min }^{-2 \tau}+\lambda^{2} u_{\min }^{-4}\right)$, where $\tau=\min \left(3-p_{1}, \ldots, 3-p_{K}, 0\right)<2$ since $1<p_{i}<5$.

For the second term in the right-hand side of (2.8), we have, by (2.6),

$$
\begin{aligned}
\int_{\Omega}|B|\left|\nabla_{g} v\right|^{2} d V_{g} & \leq C\left(\int_{\Omega}|B|^{3 / 2} d V_{g}\right)^{2 / 3}\left(\int_{\Omega}\left|\nabla_{g} v\right|^{6} d V_{g}\right)^{1 / 3} \\
& \leq C \lambda\left(\int_{\Omega}\left|\nabla_{g} v\right|^{6} d V_{g}\right)^{1 / 3} .
\end{aligned}
$$

The terms on the last line of (2.8) can be estimated as follows:

$$
\begin{aligned}
\int_{\Omega}\left|\nabla_{g} v\right|^{2} d V_{g} & =\int_{\Omega}\left|\nabla_{0} u\right|^{2} u^{-2} d x \leq C \lambda, \\
\int_{\Omega}|R|\left|\nabla_{g} v\right|^{2} d V_{g} & \leq \int_{\Omega} C\left(1+\rho(u)+\lambda u^{-4}\right)\left|\nabla_{g} v\right|^{2} d V_{g} \\
& \leq C \lambda+\left(\int_{\Omega}\left|\rho(u)+\lambda u^{-4}\right|^{3 / 2} d V_{g}\right)^{2 / 3}\left(\int_{\Omega}\left|\nabla_{g} v\right|^{6} d V_{g}\right)^{1 / 3} \\
& \leq C \lambda+C\left(\int_{\Omega}\left(\sum_{i=1}^{K} u^{(3 / 2)\left(p_{i}-5\right)+6}+\lambda^{3 / 2}\right) d x\right)^{2 / 3} \times\left(\int_{\Omega}\left|\nabla_{g} v\right|^{6} d V_{g}\right)^{1 / 3} \\
& \leq C \lambda+C \sum_{i=1}^{K} \lambda^{(1 / 4)\left(p_{i}-5\right)+1}\left(\int_{\Omega}\left|\nabla_{g} v\right|^{6} d V_{g}\right)^{1 / 3}
\end{aligned}
$$

by (1.4), since $0<\frac{3}{2}\left(p_{i}-5\right)+6 \leq 5$. (This is where we need the assumption $p_{i} \leq \frac{13}{3}$.) 
Combining all the previous estimates, we have

$$
\left(\int_{\Omega}\left|\nabla_{g} v\right|^{6} d V_{g}\right)^{1 / 3} \leq C\left(1+u_{\min }^{-2 \tau}+\lambda^{2} u_{\min }^{-4}\right) .
$$

Note that

$$
\int_{\Omega}\left|\nabla_{g} v\right|^{6} d V_{g}=\int_{\Omega}\left|\nabla_{0} u\right|^{6} u^{-18} d x
$$

So

$$
\left(\int_{\Omega}\left|\nabla_{g} u^{-2}\right|^{6} d x\right)^{1 / 3} \leq C\left(1+u_{\min }^{-2 \tau}+\lambda^{2} u_{\min }^{-4}\right) .
$$

By the Sobolev embedding theorem, for any $P, Q \in \bar{\Omega}$, we have

$$
\begin{aligned}
\left|u^{-2}(P)-u^{-2}(Q)\right| & \leq C\left\|\nabla\left(u^{-2}\right)\right\|_{L^{6}(\Omega)}|P-Q|^{1 / 2} \\
& \leq C\left\|\nabla\left(u^{-2}\right)\right\|_{L^{6}(\Omega)} \leq C\left(1+u_{\min }^{-\tau}+\lambda u_{\min }^{-2}\right) .
\end{aligned}
$$

Therefore, for any $P, Q \in \bar{\Omega}$,

$$
u^{-2}(P) \geq u^{-2}(Q)-C\left(1+u_{\min }^{-\tau}+\lambda u_{\min }^{-2}\right) .
$$

Choose $Q$ so that $u(Q)=u_{\min }=\min _{\bar{\Omega}} u$. Since $u_{\min } \leq C \lambda^{1 / 4}$ (by (1.3)) and $\tau<2$, we see that

$$
u^{-2}(Q)-C\left(1+u_{\min }^{-\tau}+\lambda u_{\min }^{-2}\right) \geq \frac{1}{2} u_{\min }^{-2},
$$

which implies that

$$
u(P) \leq C u_{\min } \leq C \lambda^{1 / 4} \text { for all } P \in \bar{\Omega} .
$$

Now let $w=u-\bar{u}$, where $\bar{u}=(1 /|\Omega|) \int_{\Omega} u$. Then $w$ satisfies

$$
\Delta w-\lambda w+f(w+\bar{u})-f(\bar{u})-\lambda \bar{u}+f(\bar{u})=0
$$

Multiplying by $w$ and integrating by parts, we get

$$
\int_{\Omega}\left(|\nabla w|^{2}+\lambda w^{2}+c(w) w^{2}\right)=0
$$

where

$$
c(w)=-\frac{f(w+\bar{u})-f(\bar{u})}{w} \rightarrow 0 \quad \text { as } \lambda \rightarrow 0 .
$$

Since $\int_{\Omega} w=0,(2.9)$ implies that $w \equiv 0$ and hence $u \equiv \bar{u}$. Theorem 1 is thus proved. 


\section{References}

[Adimurthi and Yadava 1991] Adimurthi and S. L. Yadava, "Existence and nonexistence of positive radial solutions of Neumann problems with critical Sobolev exponents", Arch. Rational Mech. Anal. 115:3 (1991), 275-296. MR 92e:35069 Zbl 0839.35041

[Adimurthi and Yadava 1993] Adimurthi and S. L. Yadava, "On a conjecture of Lin-Ni for a semilinear Neumann problem”, Trans. Amer. Math. Soc. 336:2 (1993), 631-637. MR 93f:35073 Zbl 0787.35030

[Adimurthi and Yadava 1997] Adimurthi and S. L. Yadava, "Nonexistence of positive radial solutions of a quasilinear Neumann problem with a critical Sobolev exponent", Arch. Rational Mech. Anal. 139:3 (1997), 239-253. MR 98i:35055 Zbl 0910.35050

[Chang et al. 1993] S.-Y. A. Chang, M. J. Gursky, and P. C. Yang, "The scalar curvature equation on 2- and 3-spheres”, Calc. Var. Partial Differential Equations 1:2 (1993), 205-229. MR 94k:53055 Zbl 0822.35043

[Gierer and Meinhardt 1972] A. Gierer and H. Meinhardt, "A theory of biological pattern formation”, Kybernetik (Berlin) 12 (1972), 30-39.

[Gui and Wei 2005] C. Gui and J. Wei, "On the existence of arbitrary number of bubbles for some semilinear elliptic equations with critical Sobolev exponent”, preprint, 2005.

[Keller and Segel 1970] K. F. Keller and L. A. Segel, "Initiation of slime mold aggregation viewed as an instability", J. Theor. Biol. 26 (1970), 399-415.

[Lin and Ni 1988] C. S. Lin and W.-M. Ni, "On the diffusion coefficient of a semilinear Neumann problem", pp. 160-174 in Calculus of variations and partial differential equations (Trento, 1986), edited by S. Hildebrandt et al., Lecture Notes in Math. 1340, Springer, Berlin, 1988. MR 90d:35101 Zbl 0704.35050

[Lions 1982] P.-L. Lions, Generalized solutions of Hamilton-Jacobi equations, Research Notes in Mathematics 69, Pitman, Boston, 1982. MR 84a:49038 Zbl 0497.35001

[Ni 1998] W.-M. Ni, "Diffusion, cross-diffusion, and their spike-layer steady states", Notices Amer. Math. Soc. 45:1 (1998), 9-18. MR 99a:35132 Zbl 0917.35047

[Zhu 1999] M. Zhu, "Uniqueness results through a priori estimates. I. A three-dimensional Neumann problem”, J. Differential Equations 154:2 (1999), 284-317. MR 2000c:35078 Zbl 0927.35041

Received May 15, 2001. Revised December 1, 2001.

JUNCHENG WEI

DEPARTMENT OF MATHEMATICS

Chinese University OF HONG Kong

SHATIN

HONG KONG

wei@math.cuhk.edu.hk

\section{Xingwang XU}

DEPARTMENT OF MATHEMATICS

NATIONAL UNIVERSITY OF SINGAPORE

SINGAPORE 119260

REPUBLIC OF SINGAPORE

matxuxw@nus.edu.sg 